\title{
Personalizing Antiplatelet Therapies for Acute Coronary Syndrome (ACS) in Patients Undergoing Percutaneous Coronary Intervention (PCI): Are They Cost-effective?
}

\author{
Bijan J. Borah ${ }^{1}$ - Naveen Pereira ${ }^{2}$ - Michael E. Farkouh ${ }^{3}$
}

Published online: 1 March 2017

(C) Springer Science+Business Media New York 2017

Dual antiplatelet therapy comprising of aspirin and a $\mathrm{P} 2 \mathrm{Y}_{12}$ inhibitor has been the primary treatment regimen for ACS patients undergoing PCI [1]. Prior to the introduction of prasugrel and ticagrelor, clopidogrel had been the primary antiplatelet drug used in this population. However, since there is increasing evidence that suggests limited effectiveness of clopidogrel in poor and intermediate CYP2C19 metabolizers and perhaps improved cardiovascular (CV) outcomes with the newer agents, anti-platelet drug prescription practice patterns may shift [2-4]. Following the Black Box warning by the FDA on clopidogrel, there was an added incentive for the adoption of these two newer alternatives to clopidogrel [5] Therefore, prior to adopting this change, a key policy question for health system administrators or the medical community as a whole is - whether the use of prasugrel and ticagrelor is cost-effective as compared to generic clopidogrel. Health systems are more likely to adopt these newer effective drugs when cost-effectiveness can be demonstrated. Since no head-to-head trials exist comparing the cost-effectiveness of these three drugs, the next best option would be to demonstrate the cost-effectiveness of these drugs through decision

Editorial to "CYP2C19 LOF and GOF-Guided Antiplatelet Therapy in Patients with Acute Coronary Syndrome: A Cost-Effectiveness Analysis" by Minghuan Jiang and Joyce H.S. You

Michael E. Farkouh

michael.farkouh@uhn.ca

1 Economic Evaluation Service, Kern Center for the Science of Healthcare Delivery, Mayo Clinic, Rochester, MN, USA

2 Department of Cardiovascular Diseases, Department of Molecular Pharmacology and Experimental Therapeutics, Mayo Clinic, Rochester, MN, USA

3 Peter Munk Cardiac Centre \& Heart and Stroke Richard Lewar Centre, University of Toronto, Toronto, Canada analytic modeling as performed by Jiang and You in this issue of Cardiovascular Drugs and Therapy [6].

Decision analytic models provide a framework for making decisions under uncertainty, which in this case is to estimate incremental costs and incremental effects associated with each of the three options, which in turn helps estimate the incremental cost-effectiveness ratio or ICER between pairs of options [7]. This framework provides a structured way to synthesize, use, and potentially extrapolate data for time horizons relevant for the study using a hypothetical population that resembles a real-world patient population [7]. The authors use a hybrid model - a decision-tree model for 1-year use of DAPT and a life-long Markov model for survivors of the 1year DAPT cohort to evaluate the cost-effectiveness of the three treatment strategies: (i) universal clopidogrel $75 \mathrm{mg}$ daily; (ii) universal alternative $\mathrm{P} 2 \mathrm{Y}_{12}$ inhibitor (either prasugrel $10 \mathrm{mg}$ daily or ticagrelor $90 \mathrm{mg}$ twice daily), and (iii) CYP2C19 LOF/GOF-guided therapy. Patients during the first year of DAPT may die from CV events or fatal bleeding; those that survive may remain event-free or experience nonfatal stroke, nonfatal myocardial infarction (MI), stent thrombosis, or nonfatal bleeding. Patients surviving the first year (except nonfatal MI patients) move on to the Markov model as having ischemic heart disease, and then during the yearly model cycle, patients may experience no event, acute MI, or death. The cost-effectiveness analysis was conducted from the perspective of a US healthcare provider.

The clinical inputs for the model were based on combination of randomized controlled trials and meta-analyses found from a keyword-based Medline literature search covering the period from 2000 through 2016 . The utility or disutility associated with most of the disease states in the model were from the US Medical Expenditure Panel Survey as estimated by Sullivan et al. [8]. Healthcare cost inputs for the model were derived from multiple sources including Medicare DRG rates 
for some key adverse events, commercial claims, published studies, and price-comparison websites for prescription drugs.

Patients in the CYP2C19 LOF/GOF-guided cohort experienced the lowest rates of nonfatal stroke $(0.72 \%)$, cardiovascular death (2.42\%), and major bleeding $(2.73 \%)$. Compared to universal clopidogrel and universal alternative $\mathrm{P}_{2} \mathrm{Y}_{12}$ inhibitor arms, CYP2C19 LOF/GOF-guided therapy was associated with risk reductions of 16 and $26 \%$ in nonfatal stroke, 31 and $19 \%$ in CV death, and 9 and $17 \%$ in major bleeding episodes, respectively. Patients in the universal alternative $\mathrm{P}_{2} \mathrm{Y}_{12}$ inhibitor arm had the lowest rates of nonfatal MI and stent thrombosis.

The CYP2C19 LOF/GOF-guided treatment strategy was associated with the lowest cost compared to universal clopidogrel and universal alternative $\mathrm{P} 2 \mathrm{Y}_{12}$ inhibitor therapy (\$76,450 vs. $\$ 76,906$ vs. $\$ 78,296)$, and highest QALY (7.5301 vs. 7.4381 vs. 7.4868 ), and therefore emerged as the most cost-effective treatment strategy among the three options. Compared to universal clopidogrel therapy, universal $\mathrm{P}_{2} \mathrm{Y}_{12}$ had an ICER of $\$ 28,542 / \mathrm{QALY}$, which is below the willingness-to-pay threshold of $\$ 50,000 / \mathrm{QALY}$, and therefore cost-effective. One-way sensitivity analysis evaluating the effects of parameter uncertainty one at a time indicated that universal clopidogrel becomes more cost-effective than CYP2C19 LOF/GOF-guided therapy if the hazard ratio (HR) of $\mathrm{CV}$ death in carriers versus non-carriers of CYP2C19 LOF allele among clopidogrel users is below 1.94. Similarly, if the HR of CV death in non-carriers of CYP2C19 LOF versus general clopidogrel users is less than 0.25 , universal clopidogrel emerges as the preferred strategy. Probabilistic sensitivity analysis, which assesses the uncertainty of the model results by varying all the model parameters simultaneously, was performed using 10,000 Monte Carlo simulations, CYP2C19 LOF/GOF-guided treatment strategy was found to be cost-effective $99.07 \%$ of the time, while the universal $\mathrm{P} 2 \mathrm{Y}_{12}$ inhibitor and universal clopidogrel were preferred strategies in only 0.04 and $0.89 \%$ of the time.

Jiang and You demonstrated that CYP2C19 LOF/GOFguided therapy is cost-effective compared to universal clopidogrel and universal P2Y12 inhibitor therapy [6]. However, this overall finding needs to be interpreted in the context of the study's following limitations. First, the model assumed only the perspective of a US healthcare provider. However, as suggested by the Second Panel in Costeffectiveness in Health and Medicine [9, 10], costeffectiveness analysis should be carried out both from the societal perspective and the healthcare sector perspective. The latter is important to understand the broad allocation of healthcare dollars across the population [9]. Second, model inputs can substantially influence the study results. In particular, the source of pricing information for the three antiplatelet drugs (pharmacychecker.com) considered in this study provides wide ranges and it was not clear how the authors determined the prices for these three drugs and their ranges. Similarly, with regard to the cost of clinical events, the authors simply mentioned that they obtained those costs from Medicare DRG data but it was not clear what specific MSDRGs were used, whether national or regional rates were used, and whether adjustments for wage index and geographic factors were carried out or not.

The study adds to an increasing body of evidence on the cost-effectiveness of personalized antiplatelet therapy for ACS patients undergoing PCI [11-13]. This growing evidence base will help US reimbursement authorities in making coverage decisions for personalized antiplatelet therapy since they are ultimately the gatekeepers of medical technology diffusion in the USA. The results of the NHLBI-funded TAILOR-PCI trial, the largest $\mathrm{CV}$ trial of personalized medicine, will directly address this issue [14].

\section{References}

1. Amsterdam EA, Wenger NK, Brindis RG, et al. 2014 AHA/ACC guideline for the management of patients with non-ST-elevation acute coronary syndromes: a report of the American College of Cardiology/American Heart Association Task Force on Practice Guidelines. J Am Coll Cardiol. 2014;64:e139-228.

2. Mega JL, Close SL, Wiviott SD, et al. Cytochrome P-450 polymorphisms and response to clopidogrel. New Engl J Med. 2009;360: 354-62.

3. Wallentin L, Becker RC, Budaj A, et al. Ticagrelor versus clopidogrel in patients with acute coronary syndromes. New Engl J Med. 2009;361:1045-57.

4. Wiviott SD, Braunwald E, McCabe CH, et al. Prasugrel versus clopidogrel in patients with acute coronary syndromes. New Engl J Med. 2007;357:2001-15.

5. Writing Committee Members, Holmes DR, Dehmer GJ, et al. ACCF/AHA clopidogrel clinical alert: approaches to the FDA "Boxed Warning": a report of the American College of Cardiology Foundation Task Force on Clinical Expert Consensus Documents and the American Heart Association. Circulation. 2010;122:537-57.

6. Jiang M, You J. CYP2C19 LOF and GOF-guided antiplatelet therapy in patients with acute coronary syndrome: a costeffectiveness analysis. Cardiovasc Drugs Ther. 2017;31:this issue

7. Kuntz K, Russell L, Owens D, Sanders G, Trikalinos T, Salomon JA. Decision models in cost-effectiveness analysis. In: Neumann P, Sanders G, Russel L, Siegel J, Ganiats T, editors. Cost-effectiveness in health and medicine. Second ed. New York: Oxford; 2017. pp. $105-36$.

8. Sullivan PW, Ghushchyan V. Preference-based EQ-5D index scores for chronic conditions in the United States. Med Decis Making. 2006;26(4):410-20.

9. Neumann PJ, Sanders GD. Cost-Effectiveness Analysis 2.0. N Engl J Med. 2017;376(3):203-5.

10. Sanders GD, Neumann PJ, Basu A, Brock DW, Feeny D, Krahn M, et al. Recommendations for conduct, methodological practices, and reporting of cost-effectiveness analyses: second panel on costeffectiveness in health and medicine. JAMA. 2016;316(10):1093103. 
11. Crespin DJ, Federspiel JJ, Biddle AK, Jonas DE, Rossi JS. Ticagrelor versus genotype-driven antiplatelet therapy for secondary prevention after acute coronary syndrome: a cost-effectiveness analysis. Value Health. 2011;14(4):483-91.

12. Kazi DS, Owens DK, Hlatky MA. Cost-effectiveness of genotypeguided and dual antiplatelet therapies. In response. Ann Intern Med. 2014;161(5):378-9.
13. Lala A, Berger JS, Sharma G, Hochman JS, Scott Braithwaite $\mathrm{R}$, Ladapo JA. Genetic testing in patients with acute coronary syndrome undergoing percutaneous coronary intervention: a cost-effectiveness analysis. J Thromb Haemost. 2013;11(1):8191.

14. Pereira N, Farkouh ME, et al. TAILOR-PCI. Nat Rev Cardiol. 2015;12(8):475-87. 